\title{
Low expression of IL6R predicts poor prognosis for lung adenocarcinoma
}

\author{
Gaofeng Sun ${ }^{1,2 \#}$, Tianyi Wang ${ }^{1,2 \#}$, Muqi Shi ${ }^{3}$, Hao Zhou ${ }^{1,2}$, Jinjie Wang ${ }^{1,2}$, Zhanghao Huang ${ }^{1,2}$, Haijian Zhang $^{4}$, \\ Jiahai Shi ${ }^{1,2}$
}

${ }^{1}$ Nantong Key Laboratory of Translational Medicine in Cardiothoracic Diseases, and Research Institution of Translational Medicine in Cardiothoracic Diseases, Affiliated Hospital of Nantong University, Nantong, China; ${ }^{2}$ Department of Thoracic Surgery, Affiliated Hospital of Nantong University, Nantong, China; ${ }^{3}$ Medical College of Nantong University, Nantong China; ${ }^{4}$ Research Center of Clinical Medicine, Affiliated Hospital of Nantong University, Nantong, China

Contributions: (I) Conception and design: J Shi, H Zhang; (II) Administrative support: J Shi; (III) Provision of study materials or patients: G Sun, M, H Zhou; (IV) Collection and assembly of data: T Wang, J Wang, Z Huang. (V) Data analysis and interpretation: G Sun, T Wang. (VI) Manuscript writing: All authors. (VII) Final approval of manuscript: All authors.

\#These authors contributed equally to this work.

Correspondence to: Haijian Zhang. Research Center of Clinical Medicine, Affiliated Hospital of Nantong University, Nantong 226001, China. Email: hjzhang@ntu.edu.cn; Jiahai Shi. Nantong Key Laboratory of Translational Medicine in Cardiothoracic Diseases, and Research Institution of Translational Medicine in Cardiothoracic Diseases, Affiliated Hospital of Nantong University, Nantong 226001, China. Email: sjh@ntu.edu.cn.

Background: Interleukin 6 (IL6) is both a pleiotropic cytokine and an immune-related gene. Interleukin 6 receptor (IL6R) is the receptor for IL6. It may be closely connected to the development of lung cancer. This research aims to explore the prognostic value of IL6R and prevent overtreatment of patients with lung adenocarcinoma (LUAD).

Methods: In this study, the expression of IL6R in tumor tissues and surrounding tissues was first analyzed by immunohistochemistry in the Affiliated Hospital of Nantong University (NTU) cohort. Secondly, we downloaded information from The Cancer Genome Atlas (TCGA) for the TCGA cohort and used this information to explore the messenger RNA (mRNA) level of IL6R. We then used Kaplan-Meier survival analyses, univariate and multivariate Cox analyses, nomogram models, and decision curve analyses to assess the prognostic value of IL6R. In addition, we also analyzed immune cell infiltration and the signaling pathways related to IL6R through Gene Ontology (GO), Kyoto Encyclopedia of Genes and Genomes (KEGG), and Gene Set Enrichment Analysis (GSEA).

Results: Through the data analysis of the NTU cohort and the TCGA cohort, it was found that the expression of IL6R in normal tissues around the tumor was higher than that in tumor tissue, and was positively correlated with the overall survival (OS) of LUAD patients. Additionally, low expression of IL6R was found to be an independent predictor of poor prognosis among the patients in these two research cohorts. Next, using GO, KEGG, and GSEA analyses, we found that partially infiltrated tumor immune cells might be related to earlier staging and better prognosis of patients with LUAD. Finally, the study of the 3-5-year survival rate of LUAD patients through the nomogram showed that the expression of IL6R could improve the accuracy of prediction to prevent the overtreatment of some LUAD patients.

Conclusions: In summary, our study indicated that the low expression of IL6R was associated with poor prognosis among LUAD patients and that low expression of IL6R is a potential independent risk factor that could provide a basis for strengthening postoperative classification management of such patients.

Keywords: Interleukin 6 receptor (IL6R); lung adenocarcinoma (LUAD); Kyoto Encyclopedia of Genes and Genomes (KEGG); risk factor; prognosis

Submitted Jan 04, 2021. Accepted for publication Apr 25, 2021.

doi: 10.21037/atm-21-36

View this article at: https://dx.doi.org/10.21037/atm-21-36 


\section{Introduction}

Lung cancer is recognized as the most common cancer worldwide and is also one of the main causes of death in patients with malignant tumors $(1,2)$. Lung cancer can be divided into non-small cell lung cancer (NSCLC) and small cell lung cancer, of which NSCLC (i.e., adenocarcinoma, large cell carcinoma, squamous cell carcinoma, etc.) accounts for the largest proportion of all lung cancer patients, at approximately $85-90 \%$ (3). Lung adenocarcinoma (LUAD) is the histological subtype with the highest proportion of lung cancer (40\%), followed by squamous cell carcinoma (30\%), and large cell lung cancer $(10 \%)$ (4). Although considerable progress has been made in the diagnosis and treatment of lung cancer, the 5-year survival rate of NSCLC patients after combination therapy is still very low (approximately 15\%). This is mainly due to the higher recurrence and metastasis rates of the disease $(3,5)$. Although imaging examinations are increasingly used for lung cancer patients, enabling treatment in a timely manner, the recurrence and metastasis rates of patients with NSCLC are still high. Therefore, new biomarkers to improve the prognosis of patients with LUAD are needed.

Interleukin 6 (IL6) is a versatile cytokine that plays vital roles in the proliferation and differentiation of $\mathrm{B}$ lymphocytes, $T$ lymphocytes, and hematopoietic stem cells, as well as the central nervous system, inflammation, rheumatism, autoimmune diseases, and tumor cell growth $(6,7)$. IL6 is also involved in immune regulation, inflammation, and tumorigenesis (8). However, IL6 can only conduct signal transduction by forming an IL6/ IL6R/glycoprotein 130 (gp130) hexamer complex with its receptor, thereby exerting its biological role (9-11). IL6R is an "intermediate molecule" that mediates the formation of this hexameric complex. It binds to IL6 with its corresponding structure in the extracellular domain and then further couples to the extracellular domain of gpl30 and passes through gpl30. The intracellular domain of IL6R transmits the signal of IL6 into the cell (12). The interaction of IL6 and IL6R can induce the dimerization of gp130, thereby activating signal transducer and transcription activator 3 (STAT3)(13). However, IL6/STAT3 signal activation has been shown to suppress p53-mediated miR-34a expression (13). Another study found that IL6R (rs4845626 and rs4329505) polymorphisms were associated with a reduced risk of lung cancer among smokers and nonsmokers (14), and the interaction between epithelial growth factor receptor $(E G F R)$ and IL6R in multiple tumors has been discovered. EGFR mutations and overexpression are known to contribute to the development of lung cancer, with the signaling pathways of EGFR and IL6R having a synergistic role in the occurrence and development of this disease (15). Studies have shown that the expression of IL6 is also closely related to the development of other cancers. In patients with epithelial ovarian cancer, the coexpression of EGFR and IL6R leads to a significant reduction in the overall survival (OS) rate among these patients (16). In patients with cholangiocarcinoma, IL6 causes abnormal methylation of the EGFR gene promoter, ultimately leading to malignant proliferation and apoptosis of cholangiocarcinoma cells (17). IL6R is also related to the STAT3 signaling pathway in hepatocellular carcinoma, and plays an important role in regulating cell growth and invasion (18).

So far, although the prognostic value of IL6R in patients with lung cancer has been studied (19), however, to date, little studies have evaluated the prognostic value of IL6R in patients with LUAD at different stages and its potential predictive significance in guiding the classification management of such patients after surgery. For this reason, the purpose of this study was to explore the value of IL6R in predicting the postoperative survival rates of patients with LUAD of different stages, to ultimately prevent overtreatment of lung cancer patients. Here, we explored the correlation between IL6R and the prognosis of LUAD patients of different age, gender, smoking status, and TNM stage through subgroup analysis. In addition, we confirmed the advantages of IL6R for prognosis, and constructed a new model regarding prognosis for the OS of patients with LUAD. Finally, this study also analyzed IL6Rrelated immune responses and signaling pathways through Gene Ontology (GO), Kyoto Encyclopedia of Genes and Genomes (KEGG) pathways, and Gene Set Enrichment Analysis (GSEA).

We present the following article in accordance with the REMARK reporting checklist (available at https://dx.doi. org/10.21037/atm-21-36).

\section{Methods}

\section{Study cohorts}

Two groups of LUAD patients (NTU cohort and TCGA cohort) were involved in this study. The NTU cohort included 140 patients with LUAD diagnosed by pathological paraffin sections. These 140 patients with LUAD had no 
history of chemotherapy or radiotherapy before surgery. Between 2009 and 2011, 140 LUAD samples and 34 nontumor (normal) samples were collected from lung cancer patients undergoing thoracic surgery at the First Affiliated Hospital of Nantong University, Jiangsu Province. The clinical data of these patients were retrieved from the hospital medical records archives, and included gender, age, smoking status, and tumor-lymph-node metastasis (TNM) stage. All patients underwent lung cancer staging strictly in accordance with the eighth edition of the TNM staging system (20). OS was defined as the time from randomization (in this case, surgery) to death from any cause (referring to the time of the last follow-up). The study was conducted in accordance with the Declaration of Helsinki (as revised in 2013). All patients received and signed written informed consent. This retrospective research was authorized and approved by the Clinical Research Ethics Committee of the Affiliated Hospital of Nantong University (2017-K025).

TCGA cohort was downloaded from the TCGA database (https://tcga-data.nci.nih.gov/), which was accessed on November 1, 2019 (21). The patient inclusion criteria for this study were the following: availability of clinical data (including follow-up time, survival status, TNM stage, gender, and age) and LUAD diagnosis determined by pathological paraffin sections and available messenger RNA sequencing (mRNA-seq) data on IL6R. Patients who died on the day of surgery were removed from the study. A total of 500 patients met these criteria and were selected for follow-up studies.

\section{Tissue chip, immunobistochemical staining, and evaluation of immunobistochemical staining intensity}

Formalin fixation and paraffin embedding were used to pretreat the surgical tissue samples, followed by the construction of tissue microarray (TMA) and immunohistochemistry (IHC). To construct the TMA, $2 \mathrm{~mm}$ diameter cylindrical tissue was taken from each sample, and the selected cylindrical tissue was collected into an array block using the Unitma Rapid Radiation Tissue Microarray Instrument (UT06; Unitma, Seoul, South Korea). Then, each TMA specimen was cut into $4 \mu \mathrm{m}$ tissue slices under a pathological microtome and attached to microscope slides.

IHC staining was performed as previously described (22). An anti-IL6R antibody (sc-373708; Santa Cruz Biotechnology) was used as the primary antibody (1:100 dilution). Two professional pathologists blindly assessed the proportion of IL6R positive samples and the intensity of
IL6R IHC staining. The semiquantitative immunoreactivity scoring (IRS) system was used as the assessment standard (23). Finally, we used the intensity of IL6R IHC staining (0: negative, 1: weak, 2: moderate, 3: strong) and the percentage of IL6R positive samples $(0-100)$ to calculate the semiquantitative $\mathrm{H}$-score (0-300).

\section{Immune infiltration analysis by CIBERSORT}

In order to evaluate the relationship between IL6R expression in the microenvironment of LUAD and tumorinfiltrating immune cells, we used CIBERSORT (a method for high-throughput characterization of the amount of infiltration of different cell types) to evaluate the infiltration of 21 different immune cell types from the high IL6R expression group and the low IL6R expression group (24).

\section{GO, KEGG pathway analysis, and GSEA of differentially expressed immune-related genes (DEIRGs)}

In order to analyze the functions of differential genes, the $\mathrm{R}$ package clusterProfiler (software version 3.6.3; $\mathrm{R}$ Foundation for Statistical Computing) was used for enrichment analysis of GO functional annotation and KEGG pathways. The significance threshold of a $\mathrm{P}$ value $<0.05$ was considered to indicate a significant enrichment result. In addition, we also analyzed the signaling pathways related to IL6R expression through GSEA.

\section{Statistical analysis}

Nomograms and calibration curves were plotted using $\mathrm{R}$ with the regression modeling strategies (RMS) package. The relationships between the proportions of immune cell types and survival were examined through Cox regression analysis. The differences in OS among groups were tested with the log-rank statistic using Kaplan-Meier plots. With a false discovery rate $(\mathrm{FDR}) \mathrm{P}$ value $<0.05$, the $\log$ (fold-change) $>1.0$ or $<-1.0$ defined downregulated or upregulated genes, respectively.

\section{Results}

\section{Low expression of IL6R in tumor tissue was associated with} lower survival

First, we performed IHC staining of the samples in the NTU cohort. After IHC staining, IL6R-positive staining mainly 
occurred in the cytoplasm and nucleus of LUAD and normal tissue specimens (Figure $1 A$ and Figure S1). Next, the analysis of 500 tumor samples and 21 normal tissue samples around the tumor in the TCGA cohort revealed that the expression level of IL6R mRNA in tumor tissues was lower than that in normal tissues $(\mathrm{P}<0.0001 ;$ Figure $1 B)$. We then analyzed the paired samples selected from TCGA cohort and found that the expression level of IL6R mRNA in the tissue surrounding the tumor was higher than that in the tumor tissue in the same patient $(\mathrm{P}<0.0001$; Figure $1 C)$. Next, we randomly selected 140 LUAD samples and 34 normal tissue samples around the tumor from the Affiliated Hospital of Nantong University NTU cohort and also found that the expression level of IL6R in tumor tissue was lower than that in normal tissue $(\mathrm{P}<0.0001$; Figure $1 D)$. Furthermore, in TCGA cohort $(\mathrm{P}=0.003$; Figure $1 E)$ and the NTU cohort $(\mathrm{P}=0.011$; Figure $1 F$ ), patients with high IL6R expression showed better OS than patients with low IL6R expression. It was worth noting that the IHC staining results were also consistent with the results shown in Figure $1 D$.

\section{IL6R expression in patients with LUAD was an independent risk factor}

In order to further evaluate the prognostic value of IL6R for LUAD patients, factors including IL6R expression, age, gender, smoking status, and TNM stage were sequentially incorporated into univariate and multivariate Cox regression models. The results of the univariate analysis in TCGA cohort is shown in Figure $2 A$. In the univariate analysis, the expression of IL6R $(\mathrm{P}=0.003)$, TNM stage $(\mathrm{P}<0.001)$, and $\mathrm{T}$ and $\mathrm{N}(\mathrm{P}=0.039$ and $\mathrm{P}=0.030$, respectively) were important risk factors for OS.

Subsequently, all the important risk factors affecting OS determined in the univariate analyses were used in the multivariate analyses, and the risk factors for the prognosis of LUAD patients were depicted in a forest chart. From the data analysis of TCGA cohort, IL6R expression was found to be an independent risk factor for $\mathrm{OS}$ in these LUAD patients $(\mathrm{P}=0.011)$. In addition, the TNM stage of TCGA cohort $(\mathrm{P}<0.001)$ was found to be a potentially independent risk factor for OS in these patients (Figure 2B).

The relationship between the expression of IL6R and clinical parameters among patients with LUAD in TCGA cohort is shown in Figure 2C. We selected clinical parameters related to IL6R expression from Figure $2 C$ for survival curve analysis. Notably, the higher the expression level of IL6R was, the higher the overall prognosis of the patients (Figure 2D,E, F, G,H,I). However, there were no obvious correlations between the expression of IL6R and other clinical parameters (i.e., male patients; stage II-IV patients; or M1, N1, and N2 patients). The relationships between IL6R expression and clinical parameters in patients with LUAD are also shown in Table 1.

The results of the univariate analysis in the NTU cohort are shown in Figure $3 A$. In the univariate analysis, the expression of IL6R $(\mathrm{P}=0.012)$ and TNM stage $(\mathrm{P}=0.003)$ were important risk factors for OS. Subsequently, all the important risk factors affecting OS determined in the univariate analyses were used in the multivariate analyses, and the risk factors for the prognosis of LUAD patients were depicted in a forest chart. From the data analysis of the NTU cohort ( $\mathrm{P}=0.011$; Figure 3B), IL6R expression was found to be an independent risk factor for OS in these LUAD patients. In addition, the TNM stage of the NTU cohort $(\mathrm{P}=0.004)$ was found to be a potential independent risk factor for OS in these patients. This was consistent with the analysis results in the previous NTU cohort.

The relationship between the expression of IL6R and clinical parameters among patients with LUAD in the NTU cohort is shown in Figure 3C. In the NTU cohort, IL6R expression was related to age $\leq 65(\mathrm{P}=0.022)$, non-smoker status (no) $(\mathrm{P}=0.012)$, and stage III $(\mathrm{P}=0.003)$. We then selected clinical parameters related to IL6R expression from Figure $3 B$ for survival curve analysis. Notably, the higher the expression level of IL6R was, the higher the overall prognosis of the patients (Figure 3D,E,F). However, there were no obvious correlations between the expression of IL6R and other clinical parameters (i.e., age $>65$, smoker, or stage I-II and IV patients). The relationship between IL6R expression and clinical parameters in patients with LUAD is also shown in Table 2.

\section{Comparison of prognosis of OS in patients with LUAD}

In view of the results of the univariate and multivariate analyses, a prognostic nomogram of the OS of LUAD patients was produced for TCGA cohort (Figure 4A) and the NTU cohort (Figure 4B). The nomogram based on the NTU cohort data and TCGA cohort data integrated almost all risk factors, including TNM stage, IL6R expression, gender, age, and smoking status. For each risk factor, patients obtained some points, and the total score (that is, the sum of the points obtained for each risk factor) was used to predict OS. Therefore, a higher total score was related to 

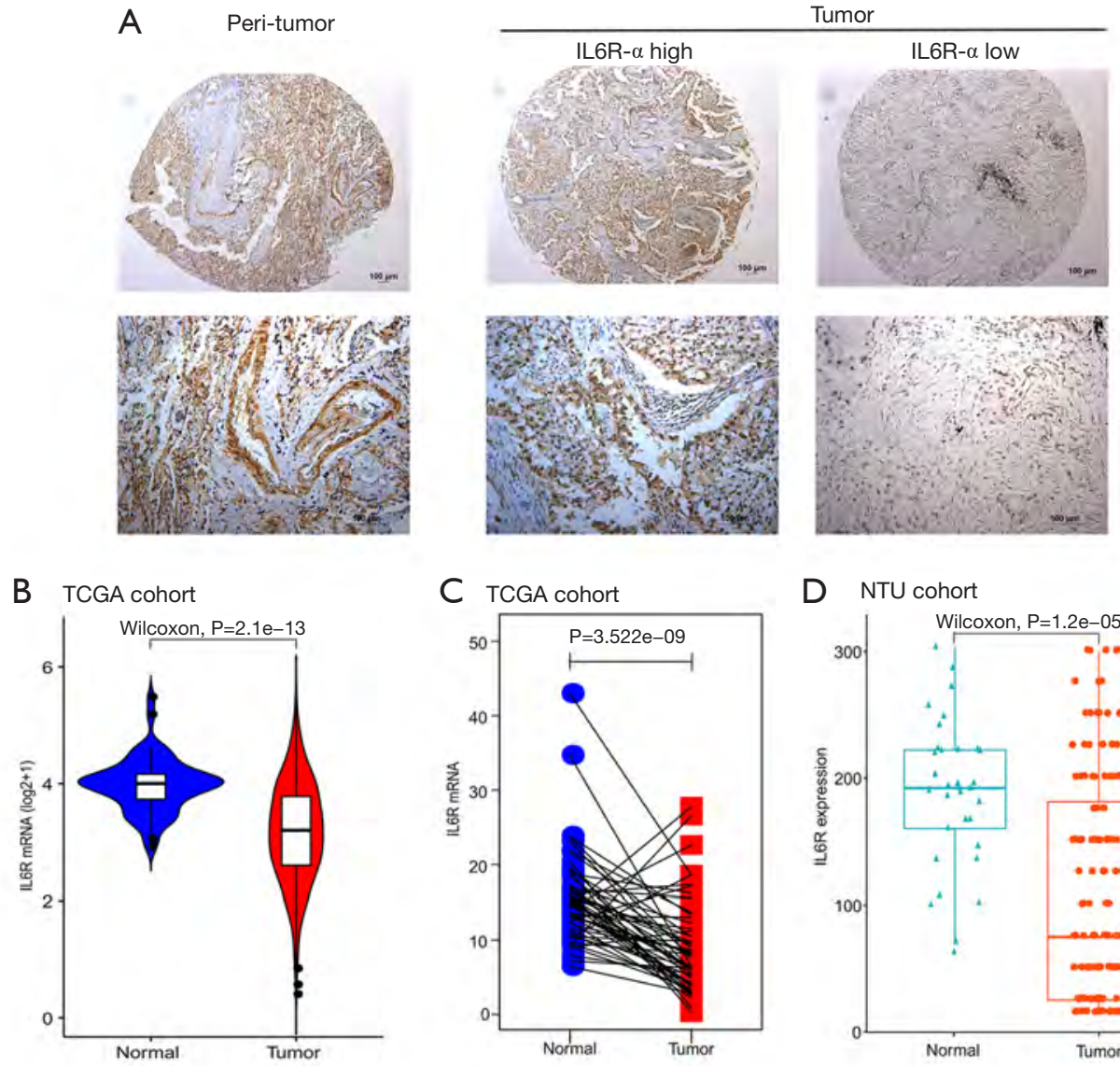

C TCGA cohort

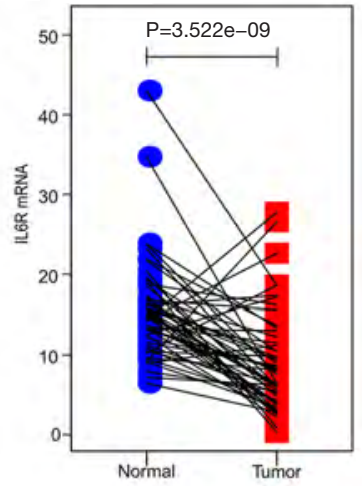

D NTU cohort
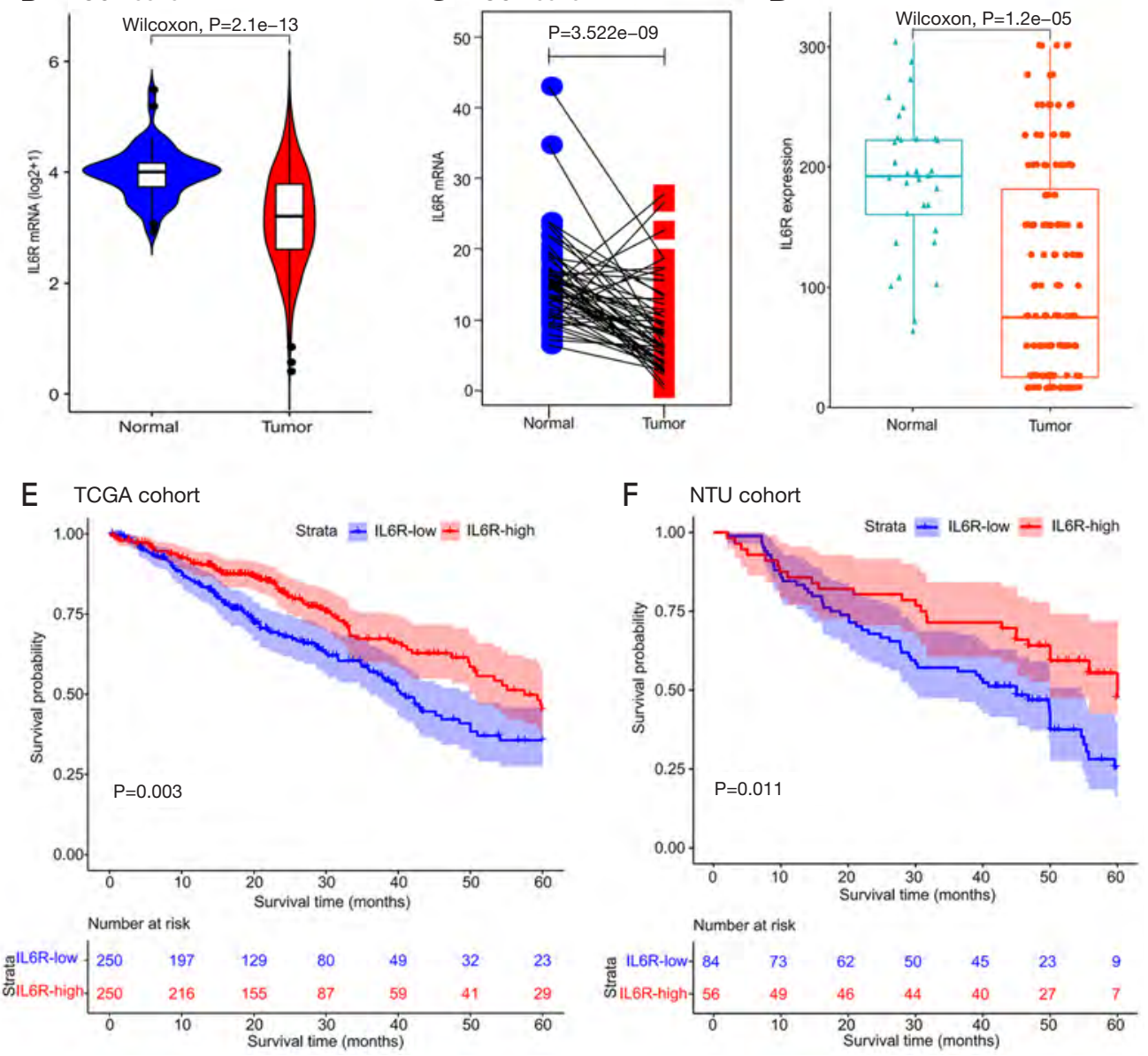

Figure 1 The expression level of interleukin 6 receptor (IL6R) in lung adenocarcinoma (LUAD) and normal tissue samples and KaplanMeier survival plots of LUAD patients. (A) Representative images of IL6R IHC were taken at 5x (top) and 20× (bottom) magnifications. The scale bars are marked in the lower right corner of the image; (B) IL6R mRNA levels in the tumor and surrounding tissues of The Cancer Genome Atlas (TCGA) cohort; (C) IL6R mRNA levels in the paired samples of TCGA cohort; (D) the staining intensity of IL6R IHC in the tumor and adjacent tissues of the Affiliated Hospital of Nantong University (NTU) cohort; (E,F) the survival curve of the high and low expression of IL6R in 500 LUAD patients in TCGA cohort and the survival curve of the high and low expression of IL6R in 140 LUAD patients in the NTU cohort. 


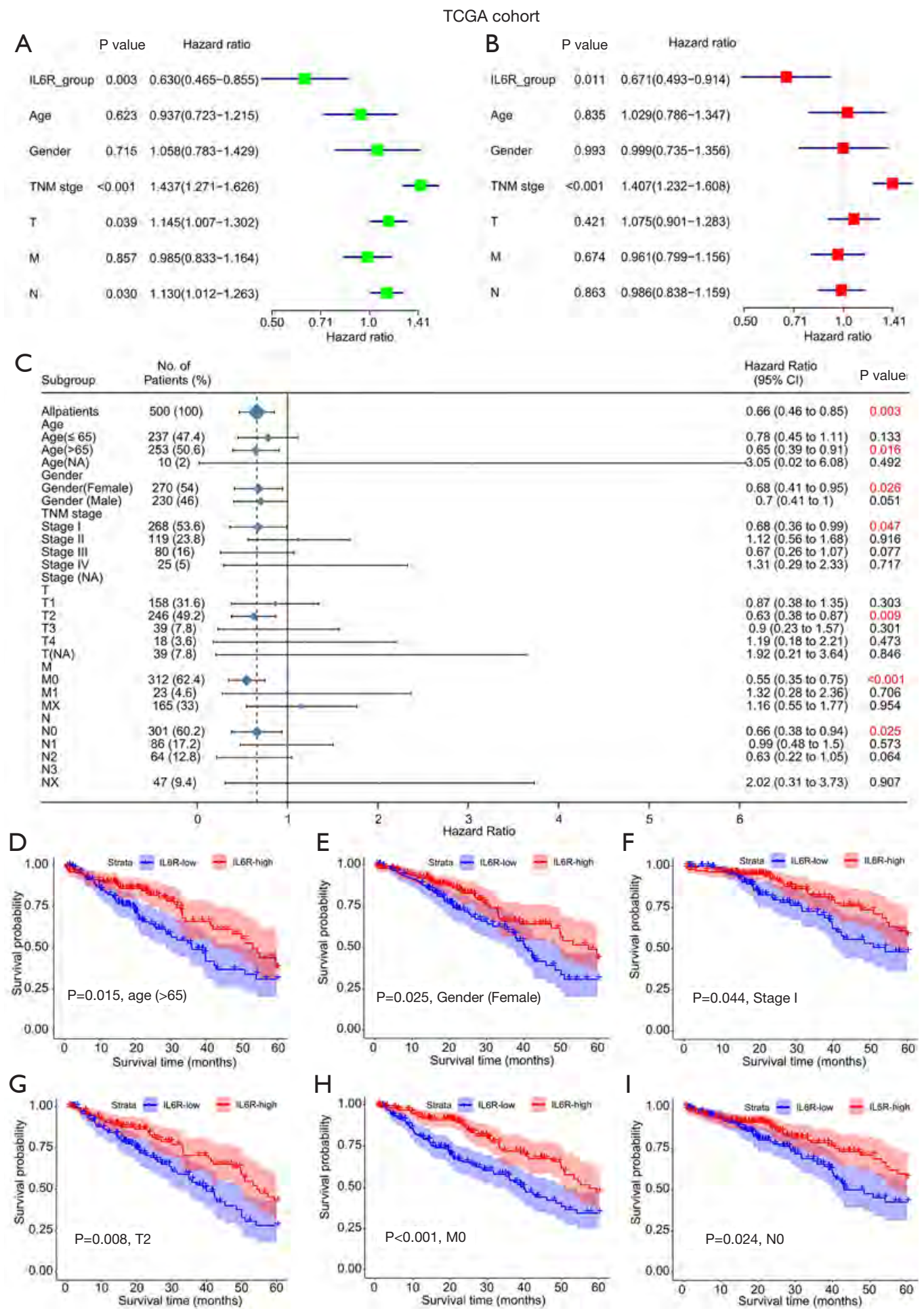

Figure 2 The relationship between interleukin 6 receptor (IL6R) expression and clinical parameters in patients with lung adenocarcinoma (LUAD) in the univariate and multivariate analyses and its influence on the overall survival of patients. (A) Single factor analysis and (B) multivariate analysis of the relationship between IL6R expression and clinical parameters in LUAD patients in TCGA cohort; (C) the relationship between IL6R expression and various clinical parameters; (D,E,F,G,H,I) survival curves of LUAD patients with different clinical parameters and high and low expression of IL6R. 
Table 1 The relationship between IL6R expression and clinical parameters in patients with LUAD in TCGA cohort

\begin{tabular}{|c|c|c|c|c|}
\hline Variables & IL6R low (n=250) & IL6R high $(n=250)$ & Total $(n=500)$ & $P$ value \\
\hline$\leq 65$ & $125(50.0)$ & $112(44.8)$ & $237(47.4)$ & \\
\hline$>65$ & $122(48.8)$ & $131(52.4)$ & $253(50.6)$ & \\
\hline NA & $3(1.2)$ & $7(2.8)$ & $10(2.0)$ & 0.268 \\
\hline Female & $122(48.8)$ & $148(59.2)$ & $270(54.0)$ & \\
\hline Male & $128(51.2)$ & $102(40.8)$ & $230(46.0)$ & 0.025 \\
\hline \multicolumn{5}{|c|}{ Stage, n (\%) } \\
\hline I & $121(48.4)$ & $147(58.8)$ & $268(53.6)$ & \\
\hline IV & $10(4.0)$ & $15(6.0)$ & $25(5.0)$ & \\
\hline NA & $3(1.2)$ & $5(2.0)$ & $8(1.6)$ & 0.003 \\
\hline \multicolumn{5}{|l|}{$\mathrm{T}, \mathrm{n}(\%)$} \\
\hline $\mathrm{T} 1$ & $70(28.0)$ & 88 (35.2) & $158(31.6)$ & \\
\hline $\mathrm{T} 2$ & $125(50.0)$ & $121(48.4)$ & 246 (49.2) & \\
\hline T3 & $25(10.0)$ & $14(5.6)$ & $39(7.8)$ & \\
\hline $\mathrm{T} 4$ & $8(3.2)$ & $10(4.0)$ & $18(3.6)$ & \\
\hline NA & $22(8.8)$ & $17(6.8)$ & $39(7.8)$ & 0.193 \\
\hline \multicolumn{5}{|l|}{ M, n (\%) } \\
\hline No & $142(56.8)$ & $159(63.6)$ & $301(60.2)$ & \\
\hline $\mathrm{N} 1$ & $40(16.0)$ & $46(18.4)$ & $86(17.2)$ & \\
\hline N2 & $43(17.2)$ & $21(8.4)$ & $64(12.8)$ & \\
\hline N3 & $2(0.8)$ & $0(0)$ & $2(0.4)$ & \\
\hline NA & $23(9.2)$ & $24(9.6)$ & $47(9.4)$ & 0.019 \\
\hline
\end{tabular}

IL6R, interleukin 6 receptor; LUAD, lung adenocarcinoma; TCGA, The Cancer Genome Atlas; NA, not applicable.

higher risk and poor prognosis. For TCGA (Figure 4C,D) and NTU (Figure 4E,F) cohorts, the calibration curve of the 3- or 5-year survival probabilities between the predicted results of the nomogram and the actual observations showed a strong correlation.
The relationship between IL6R expression and tumorinfiltrating immune cells

To screen differentially expressed genes, we used the median expression of IL6R to classify the gene expression profiles of TCGA cases and found two sets of differential 

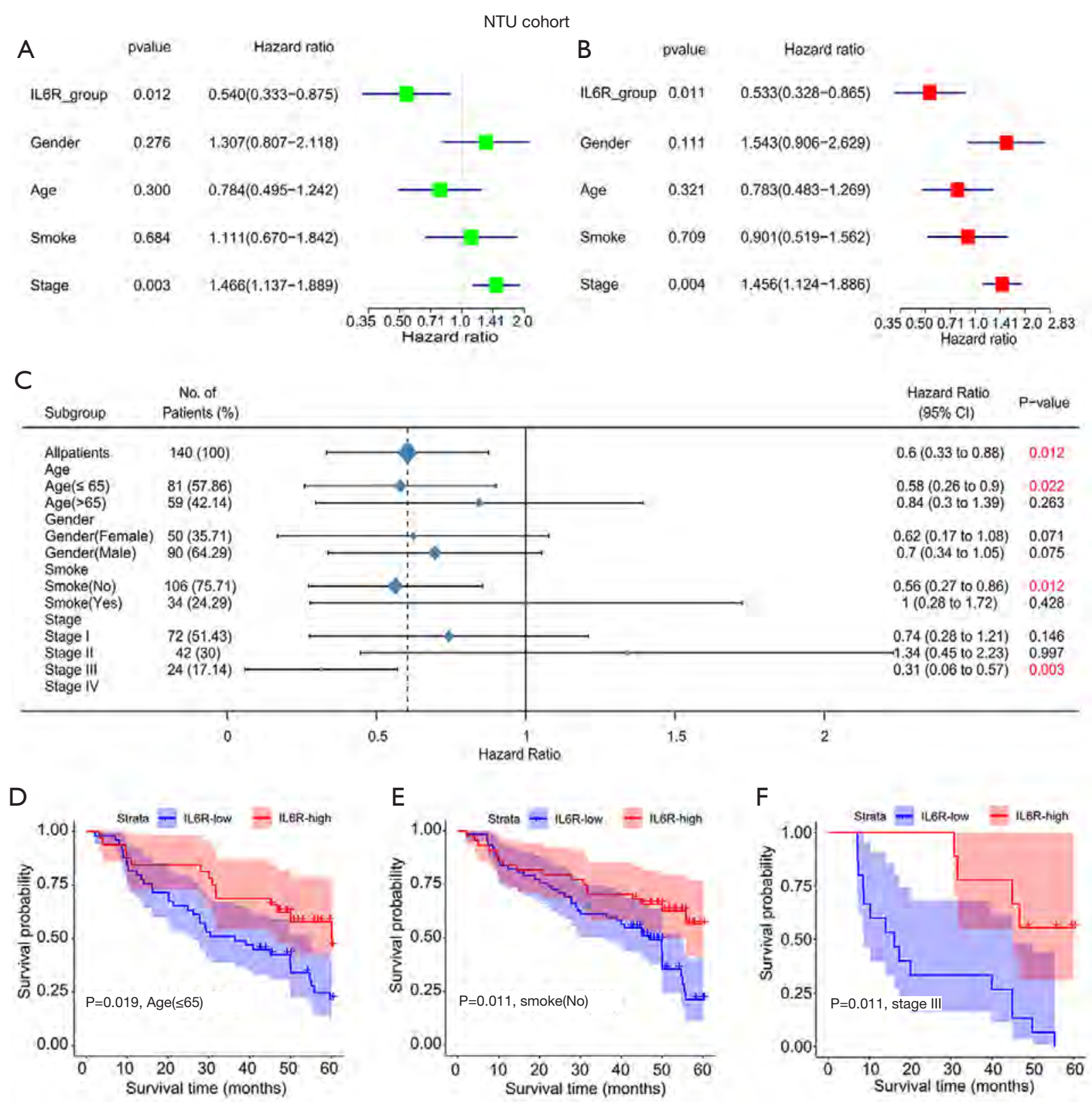

Figure 3 Single-factor and multivariate analyses of the relationship between interleukin 6 receptor (IL6R) expression and clinical parameters in lung adenocarcinoma (LUAD) patients and its impact on the overall survival of patients. (A) Single-factor and (B) multivariate analyses of the relationship between IL6R expression and clinical parameters in LUAD patients in the NTU cohort; (C) the relationship between IL6R and various clinical parameters; (D,E,F) survival curves of LUAD patients with different clinical parameters and high and low expression of IL6R.

genes, as shown in Figure S2A,B. We aimed to determine whether the tumor immune microenvironment of LUAD patients with high IL6R expression was different from that of LUAD patients with low IL6R expression. According to the expression of IL6R, 500 tumor samples were divided into two groups, with 250 tumor samples in the high expression group and 250 tumor samples in the low expression group. We used the established computing resource, CIBERSORT, to explore the gene expression profile of the downloaded sample to determine the levels of 
Table 2 The relationship between IL6R expression and clinical parameters in patients with LUAD in NTU cohort

\begin{tabular}{|c|c|c|c|c|}
\hline Variables & IL6R low $(\mathrm{n}=84)$ & IL6R high ( $\mathrm{n}=56$ ) & Total $(n=140)$ & $P$ value \\
\hline \multicolumn{5}{|l|}{ Sex, n (\%) } \\
\hline Female & $31(36.9)$ & 19 (33.9) & $50(35.7)$ & \\
\hline Male & $53(63.1)$ & $37(66.1)$ & $90(64.3)$ & 0.857 \\
\hline \multicolumn{5}{|c|}{ Age (years), n (\%) } \\
\hline$\leq 65$ & $49(58.3)$ & $32(57.1)$ & 81 (57.9) & \\
\hline$>65$ & $35(41.7)$ & 24 (42.9) & 59 (42.1) & 1 \\
\hline \multicolumn{5}{|c|}{ Smoke, n (\%) } \\
\hline No & $62(73.8)$ & 44 (78.6) & $106(75.7)$ & \\
\hline Yes & $22(26.2)$ & $12(21.4)$ & $34(24.3)$ & 0.658 \\
\hline \multicolumn{5}{|c|}{ Stage, n (\%) } \\
\hline I & $39(46.4)$ & 33 (58.9) & $72(51.4)$ & \\
\hline$\|$ & $28(33.3)$ & $14(25.0)$ & $42(30.0)$ & \\
\hline III & $15(17.9)$ & $9(16.1)$ & $24(17.1)$ & \\
\hline IV & $2(2.4)$ & $0(0)$ & $2(1.4)$ & 0.394 \\
\hline
\end{tabular}

IL6R, interleukin 6 receptor; LUAD, lung adenocarcinoma.

21 immune cells. The CIBERSORT algorithm applied to 21 immune cell subtypes helped to evaluate the differences in immune cell abundance levels between the high and low IL6R expression groups (Figure 5). The infiltration of memory B cells $(\mathrm{P}=0.012)$, resting memory $\mathrm{CD} 4 \mathrm{~T}$ cells $(\mathrm{P}<0.001)$, monocytes $(\mathrm{P}<0.001), \mathrm{M} 2$ macrophages $(\mathrm{P}=0.011)$, resting dendritic cells $(\mathrm{P}<0.001)$, activated dendritic cells $(\mathrm{P}=0.002)$, resting mast cells $(\mathrm{P}<0.001)$, and activated mast cells $(\mathrm{P}=0.004)$ in LUAD patients with high IL6R expression was high, while the infiltration of these cells in the low IL6R expression group was low. However, the infiltration of activated memory CD4 T cells $(\mathrm{P}=0.001)$, M0 macrophages $(\mathrm{P}<0.001)$, and $\mathrm{M} 1$ macrophages $(\mathrm{P}=0.033)$ in LUAD patients with low IL6R expression was higher than the infiltration in the high IL6R expression group. Significant differences were observed in resting memory CD4 T cells, monocytes, M0 macrophages, resting dendritic cells, and resting mast cells between the high and low IL6R expression groups. Compared with the low IL6R expression group, the infiltration levels of resting memory CD4 $\mathrm{T}$ cells, monocytes, resting dendritic cells, and resting mast cells in the high IL6R expression group were significantly increased $(\mathrm{P}<0.001)$. However, compared with the low IL6R expression group, the infiltration level of M0 macrophages in the high expression group was significantly reduced $(\mathrm{P}<0.001)$. We also assessed the possible correlations between the 21 types of immune cells (Figure S3). The generated heat map showed that the ratio of part of the tumor-infiltrating immune cell subsets had a strong correlation. Among them, gammadelta $\mathrm{T}$ cell had the greatest positive correlation with CD8 $\mathrm{T}$ cell (Corr $=1$ ). M0 macrophage had the largest negative correlation with resting mast cell (Corr $=-0.4)$, and resting memory CD4 $\mathrm{T}$ cells also had the largest negative correlation with CD8 $T$ cells (Corr $=-0.4)$. In order to further explore the relationship between IL6R and the immune system. For example, to evaluate the impact of key immune system markers in survival analysis to take into account the level of IL6R. We conducted statistical analysis and found that among the 11 immune cells associated with IL6R found in the previous analyses of this study, M0 macrophages cells and resting dendritic were significantly associated with the 5 -year OS of patients with LUAD. The remaining immune cells had no significant correlation with the prognosis of LUAD. Among them, the expression level of M0 macrophages was negatively correlated with the 5 -year overall prognosis of patients with LUAD $(\mathrm{P}=0.012$; Figure $6 \mathrm{~A}$ ), while resting dendritic cells was positively correlated with the 5 -year overall prognosis of patients 

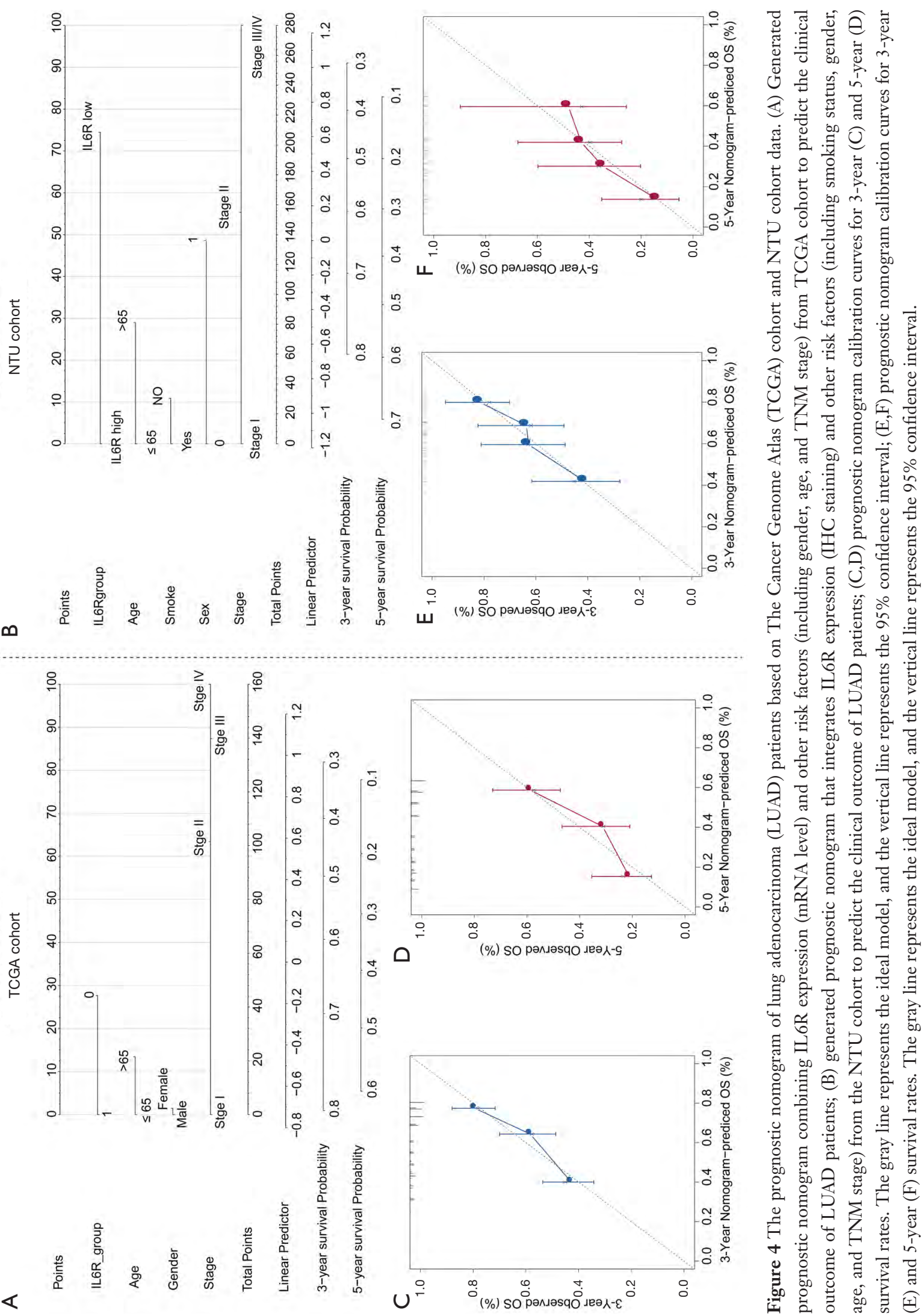
with LUAD ( $\mathrm{P}=0.043$; Figure $6 B)$.

Next, we divided LUAD patients into two different clusters (high and low immune infiltration groups) according to the level of immune cell infiltration. We then analyzed the relationships between different immune infiltration groups and the level of IL6R expression and other clinical parameters. We found that IL6R expression was positively correlated with tumor-infiltrating immune cells, and the more tumor immune cell infiltration, the earlier the clinical stage and the higher the 5 -year OS. The results were shown in Figure 7.

\section{The differential expression of EGFR and KRAS in LUAD patients in the IL6R high and low expression group and its effect on the OS of LUAD patients}

In order to explore the relationship between IL6R and the lung cancer driver genes EGFR and KRAS and its impact on the OS of LUAD patients in the high and low IL6R expression groups, we analyzed the differential expression of EGFR and KRAS in LUAD patients with high and low IL6R expression groups and analyzed the effects of $E G F R$ and KRAS on the OS of LUAD patients with high and low IL6R expression groups. The results showed that the expression of EGFR in LUAD patients with low IL6R expression was significantly higher than that in LUAD patients with high IL6R expression $(\mathrm{P}<0.001$; Figure $8 A)$. However, no significant difference in $K R A S$ expression was observed in patients with LUAD with high and low IL6R expression (Figure 8B). Then combined with the expression level of IL6R and the Kaplan-Meier curve of the expression levels of lung cancer driver genes EGFR and $K R A S$, the results showed that $E G F R$ and KRAS had no significant difference in the survival of LUAD patients between the IL6R high and low expression groups (Figure 8C,D).

\section{GO, KEGG pathway analysis, and GSEA of DEIRGs}

In order to further understand the functions of DEIRGs, we used the cluster Profiler software package in R to carry out functional and pathway enrichment analysis of DEIRGs. The results of GO analysis showed that the biological processes were enriched in neutrophil-mediated immunity, neutrophil activation involved in immune response, regulation of innate immune response, and positive regulation of innate immune response (Figure 9A). KEGG pathway analysis showed that DEIRGs were mainly related to several immune-related signaling pathways (Figure 9B).
GSEA showed that the two pathways positively related to IL6R expression were the intestinal immune network for immunoglobin $\mathrm{A}(\mathrm{IgA})$ production $(\mathrm{P}=0.007)$ and Th17 cell differentiation $(\mathrm{P}=0.019)$. The three pathways with the strongest negative correlation were the p53 signaling pathway $(\mathrm{P}=0.011)$, Staphylococcus aureus infection $(\mathrm{P}=0.015)$, and the IL17 signaling pathway $(\mathrm{P}=0.026)$, as shown in Figure 9C.

\section{Discussion}

Lung cancer is the most common cause of cancer-related deaths worldwide (25). The development of EGFR tyrosine kinase inhibitors (TKIs) has changed the clinical management of lung cancer (25). However, the war on lung cancer is still far from over. In this study, it was found that the expression of IL6R in LUAD patients was significantly correlated with OS, especially in clinical stage III patients. Patients with high IL6R expression had a better prognosis than patients with low IL6R expression.

IL6 is an important cytokine with multiple functions, including regulating cell growth, differentiation, and participating in immune responses (26-28). In the liver, it has been shown to stimulate the expression of acute phase proteins SAA and CRP (29). IL6R is the receptor of IL6 and exists in two forms, namely mIL6R and sIL6R (30). The sIL6R was initially identified as a receptor involved in IL6 anti-signal transduction, and was later identified as an antiviral protein independent of IL6 (31-33). The genetic polymorphism of IL6 and/or IL6R is related to respiratory diseases, autoimmune diseases, and several types of cancer $(27,34)$. According to reports, the $\mathrm{C}$ allele of rs 2228145 in IL6R was associated with a decrease in serum IL6R levels and lung function in asthma patients (35). Studies have also shown that there is a correlation between rs2228145 and the risk of rheumatoid arthritis in the Pakistani population (36). In addition, it was reported that rs 2228145 had a protective effect on gastric cancer risk in patients without Helicobacter pylori infection (8). An increasing amount of evidence has shown that sIL6R forms a complex with sgp130/IL6ST in the circulation to inhibit IL6 (37).

At present, a large number of studies have confirmed that IL6R is related to tumors $(15-18)$. Studies have even shown that IL6 could be used as a prognostic biomarker for cholangiocarcinoma, and IL6R could be used as a therapeutic target for cholangiocarcinoma (38). However, there are few studies on IL6R in LUAD. Therefore, we conducted this study based on available data to explore 


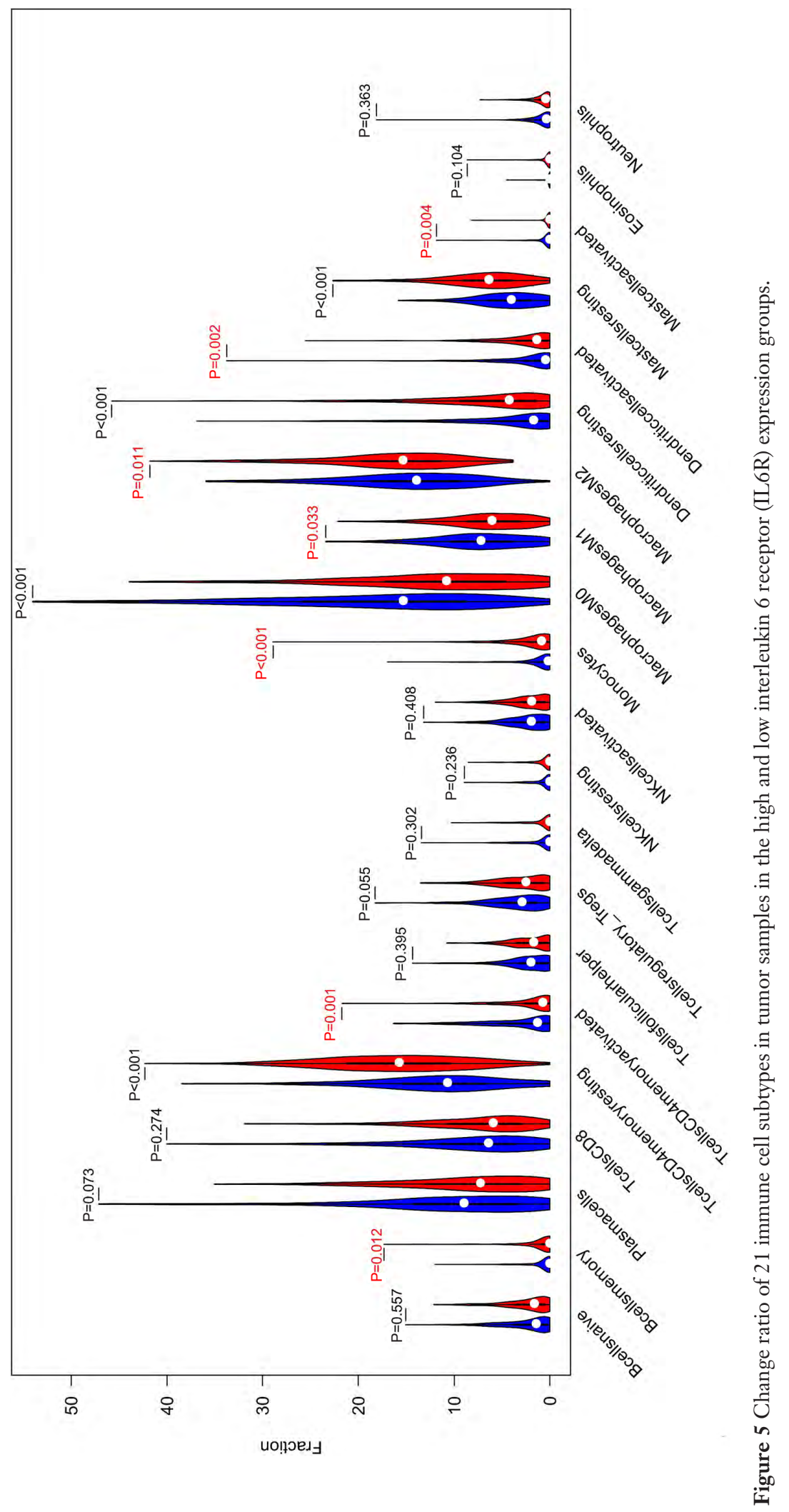




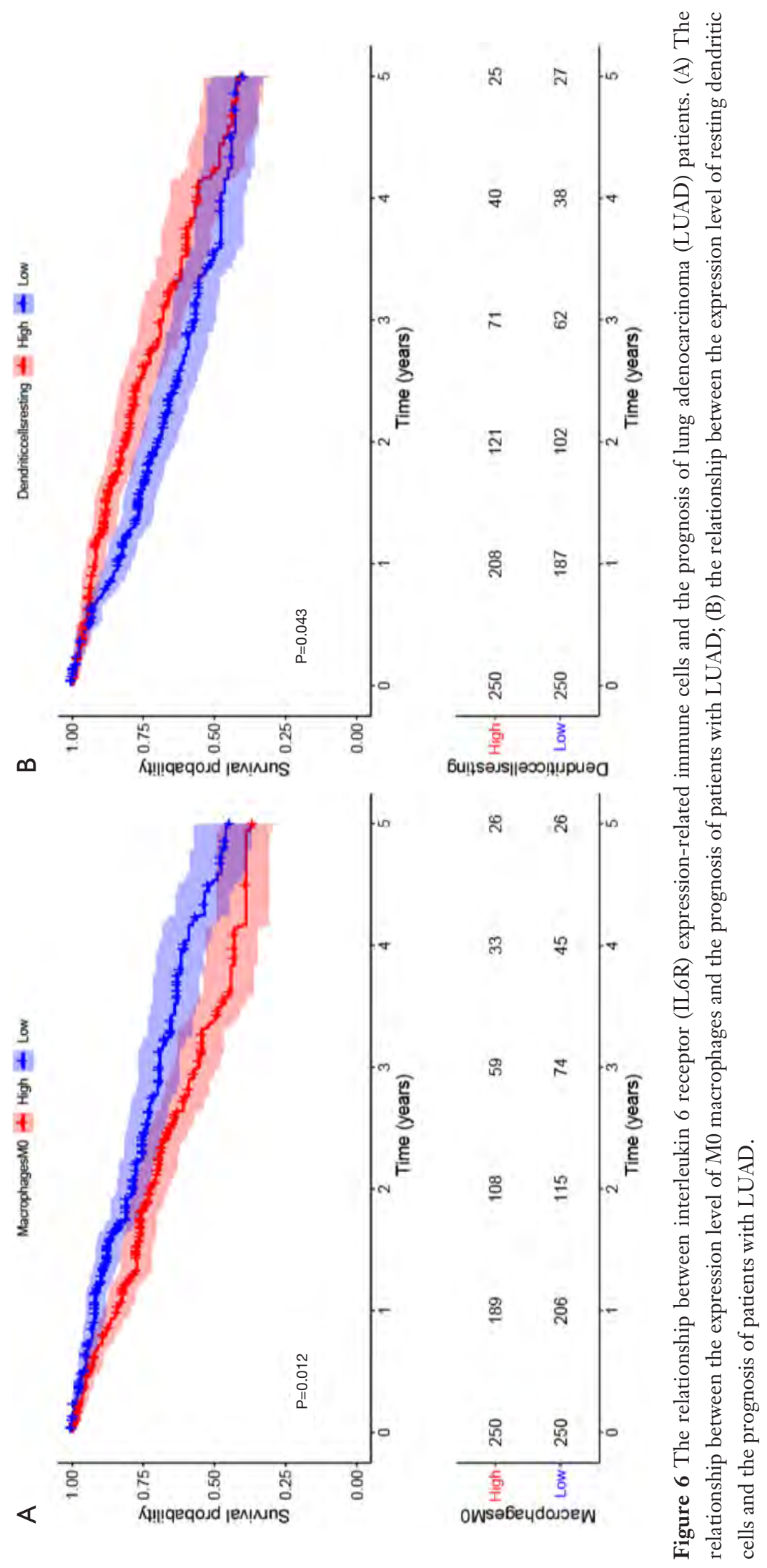




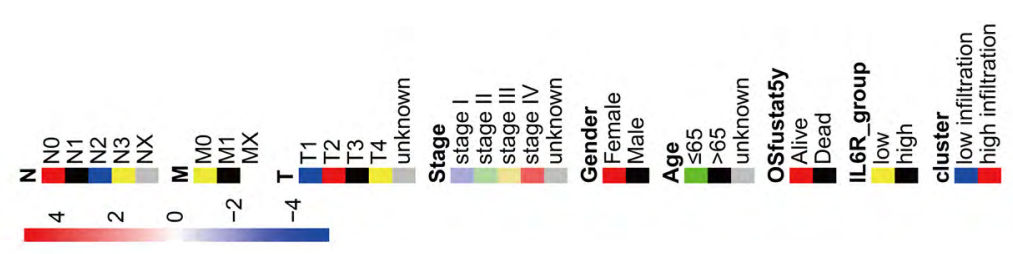

吾
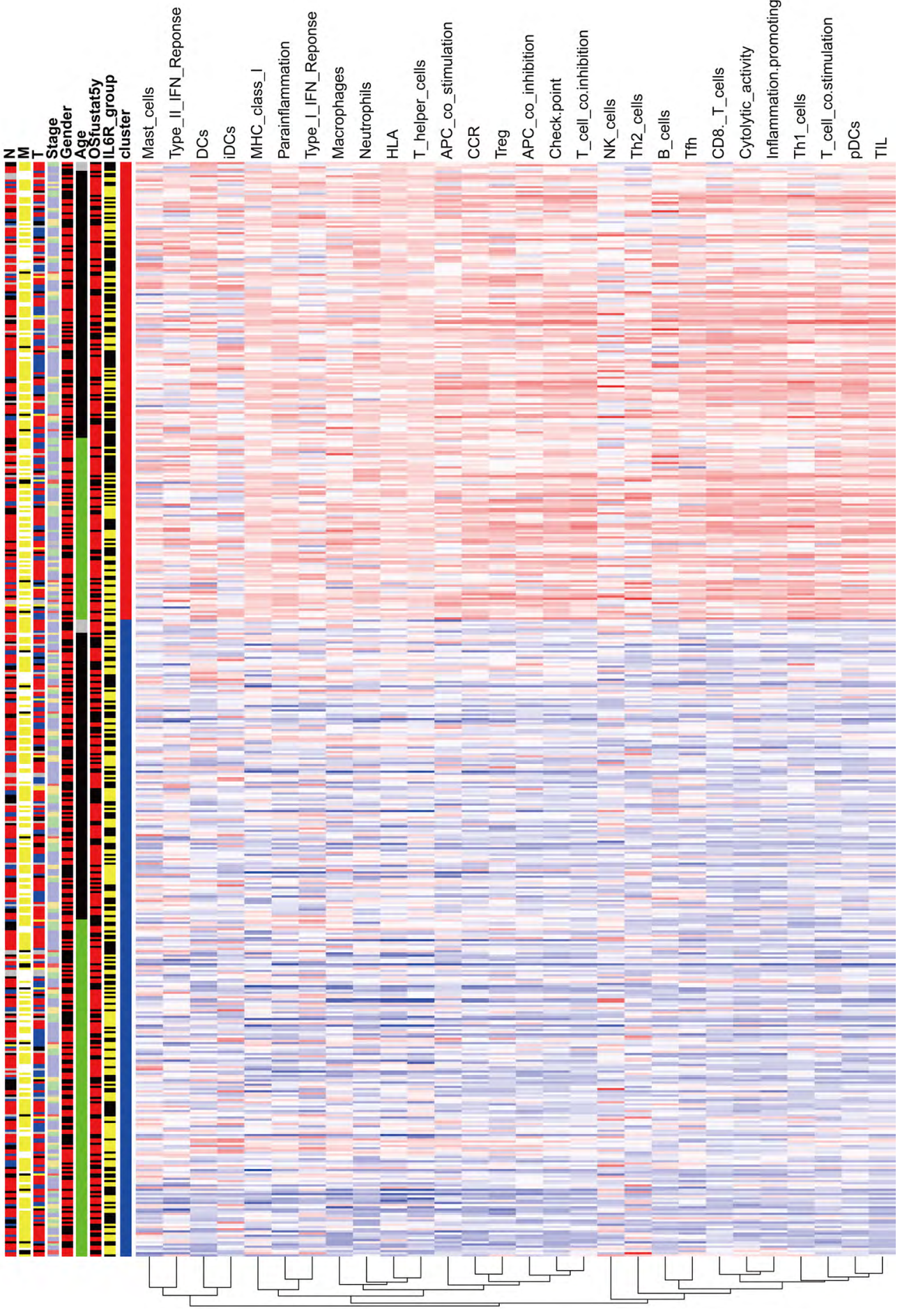

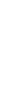

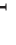

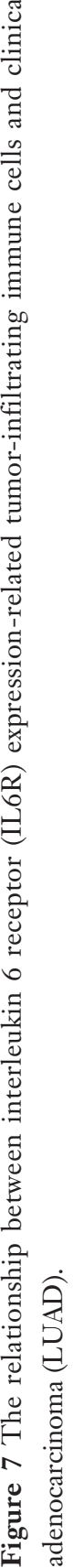




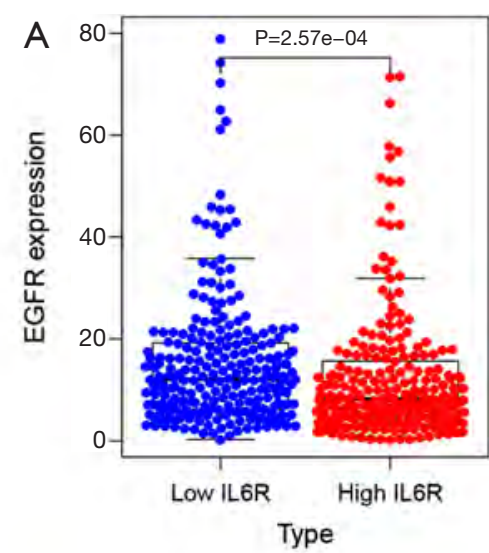

C

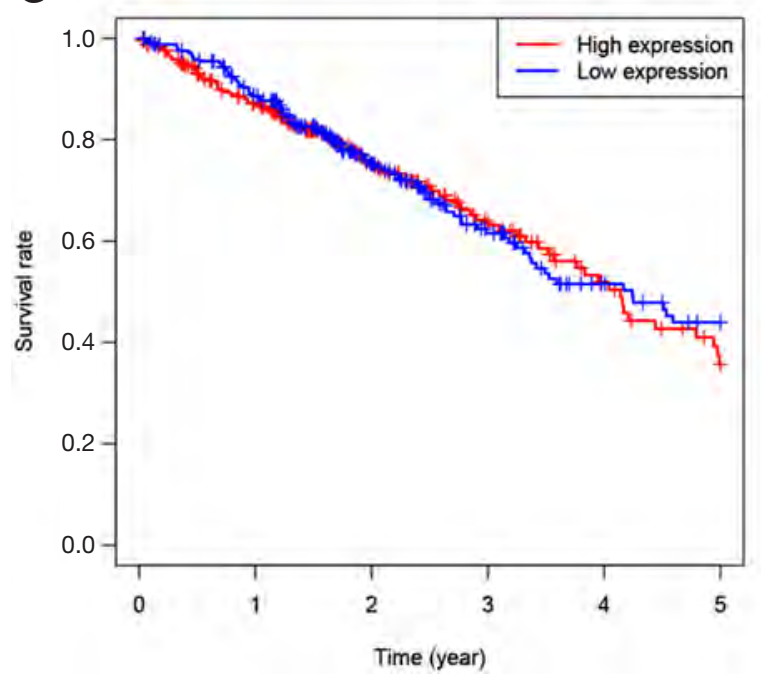

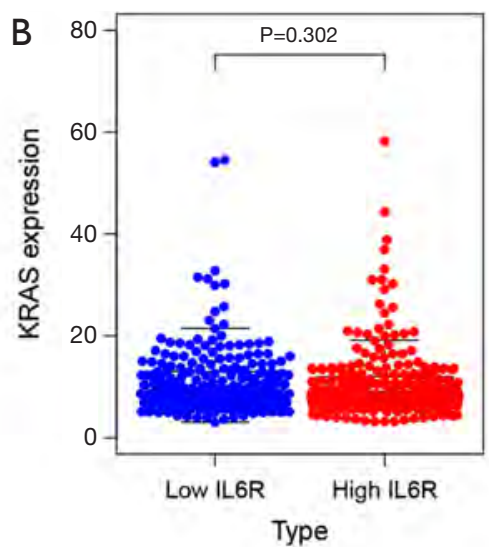

D

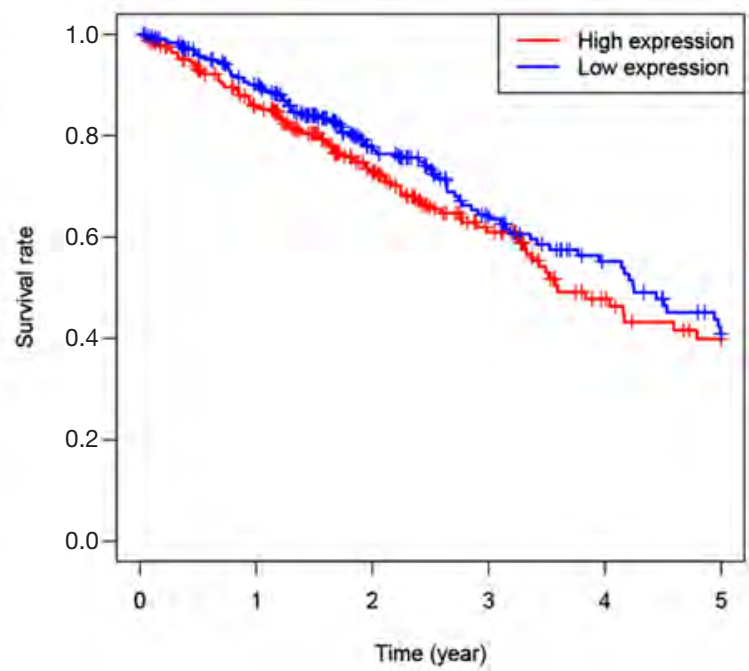

Figure 8 The differential expression of EGFR and KRAS in lung adenocarcinoma (LUAD) patients in the high and low interleukin 6 receptor (IL6R) expression group and its effects on the OS of LUAD patients. (A) The differential expression of $E G F R$; (B) the differential expression of $K R A S$; (C) effects of EGFR on OS of patients with LUAD in high and low IL6R expression groups; (D) effects of KRAS on OS of patients with LUAD in high and low IL6R expression groups.

the prognostic value of IL6R in patients with LUAD. Our study found that IL6R expression was significantly related to TNM stage and OS in patients with LUAD. In addition, GO, KEGG, and GSEA analyses showed that IL6R expression was closely related to tumor immune responses and certain signaling pathways, such as the IL17 signaling pathway and the p53 signaling pathway, amongst others. The IL17 molecular cytokine family includes 6 members, namely IL17A, IL17B, IL17C, IL17D, IL17E, and IL17F. At present, research on IL17 has been carried out in many tumor types. For example, IL17A was found to promote the proliferation of intestinal epithelial cells with deletion mutation of the tumor suppressor gene, APC, and promote the occurrence of intestinal cancer (39). In a pancreatic epithelial tumor model with Kras mutation, Kras mutation could induce pancreatic epithelial cells to express the IL17A receptor and recruit IL17A-expressing immune cells; furthermore, the overexpression of IL17A significantly accelerated the initiation and development of pancreatic epithelial cell tumors (40). IL17B has also been reported to be expressed in a variety of breast cancer cell lines and human breast cancer pathological samples (41). Knockdown of IL17B expression in breast cancer cell lines inhibited the proliferation and invasion of these cells (41). However, 
A

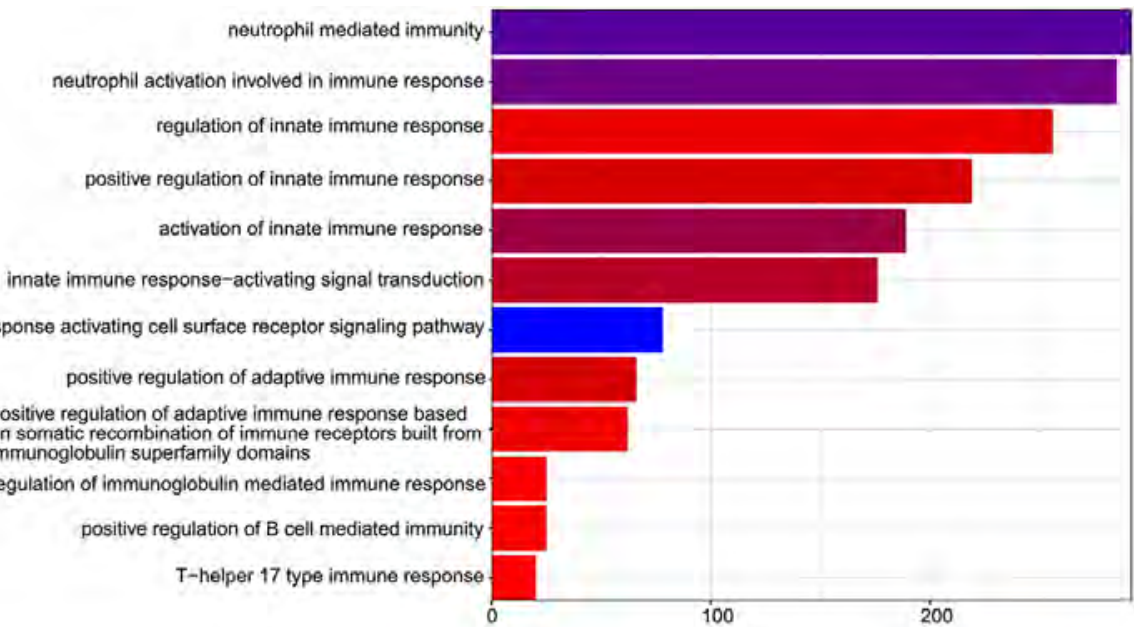

B

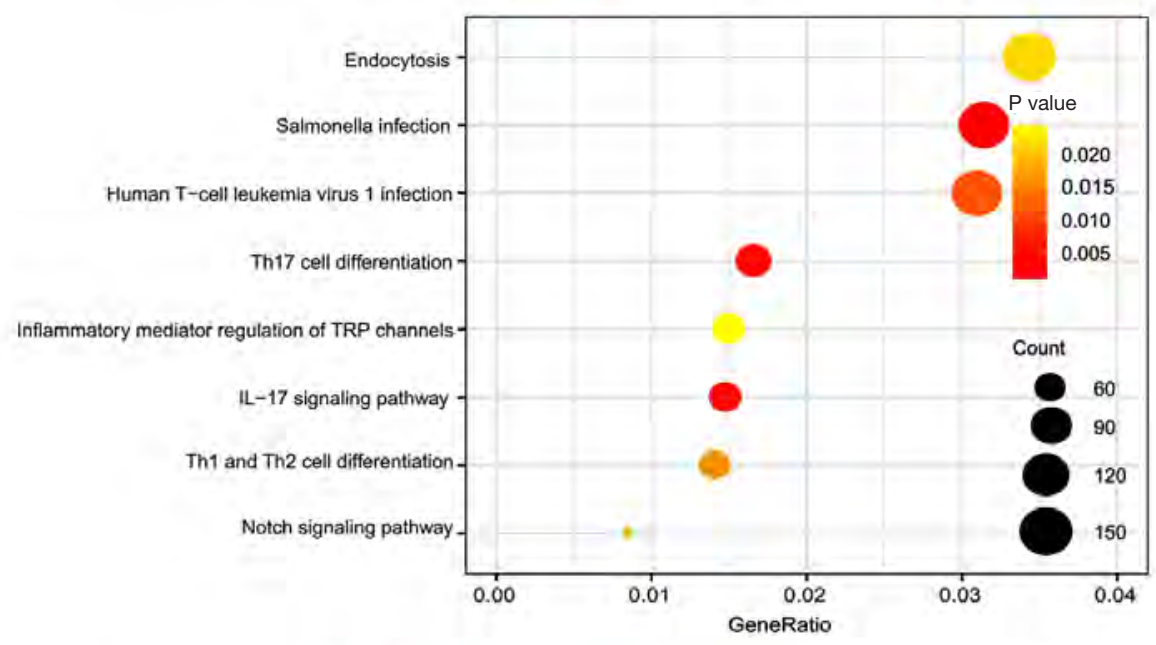

C

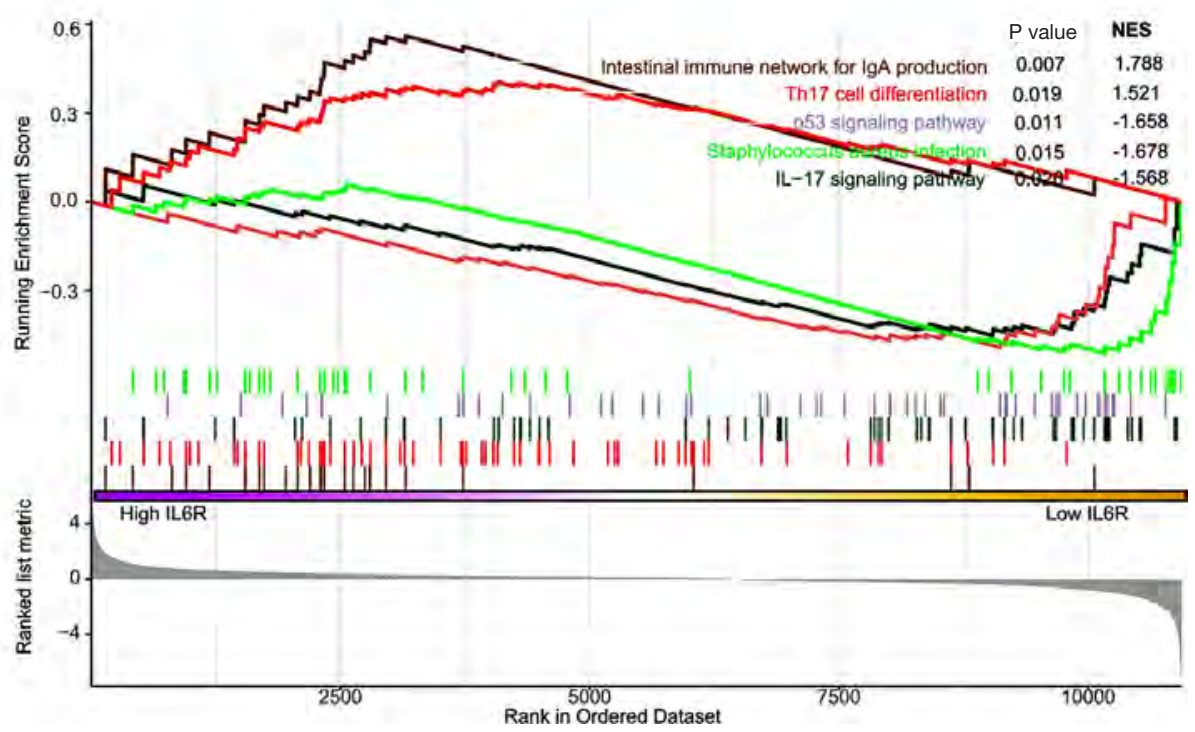

Figure 9 Gene Ontology (GO), Kyoto Encyclopedia of Genes and Genomes (KEGG) pathway analysis, and Gene Set Enrichment Analysis (GSEA) of differentially expressed immune-related genes (DEIRGs). (A) GO analysis of DEIRGs; (B) KEGG pathway enrichment analysis of DEIRGs; (C) signaling pathways related to IL6R expression determined by GSEA. 
from the current point of view, there are few studies on the mechanism of IL17 in LUAD and the relationship between IL6R and IL17 signaling pathways in LUAD. Therefore, GSEA provided a basis for us to further study the mechanism of IL17 in LUAD and the relationship between IL6R and IL17 signaling pathways in LUAD. Overall, these results demonstrate that the expression of IL6R may have important clinical significance for the prognosis of patients with LUAD.

Further work is needed to strengthen our research results. First, recent and ongoing oncology studies have attempted to establish correlations between disease-free survival, progression-free survival, and other outcomes or risk factors. Owing to the lack of follow-up information on disease recurrence and disease progression in LUAD patients, this research only described the correlation analysis between IL6R expression and OS. Establishing further correlations will require additional work, and our future research will focus on filling these gaps, particularly in terms of disease recurrence and progression. Second, the influence of IL6R expression on the prognosis of LUAD patients should be further verified in a prospective multicenter clinical trial, which is essential to enhance the reliability of the prognostic map on the basis of IL6R expression. Third, the expression level of IL6R has not been studied in suspected LUAD patients, which we will explore in future research. Finally, the specific role of IL6R in the occurrence and development of LUAD is still unclear, and further studies are needed to clarify its underlying mechanism.

In conclusion, this study determined that IL6R can predict unsatisfactory prognosis among patients with LUAD, and the detection of its expression level can enhance the postoperative management of patients with LUAD in different TNM stages. In addition, our prognostic nomogram based on IL6R expression and other risk factors significantly improves the accuracy of patient survival prediction and can prevent overtreatment of patients with LUAD.

\section{Acknowledgments}

We thank the help of all the researchers from the Nantong Key Laboratory of Translational Medicine in Cardiothoracic Diseases, the Nantong Clinical Medical Research Center of Cardiothoracic Disease, and the Institution of Translational Medicine in Cardiothoracic Diseases in Affiliated Hospital of Nantong University.
Funding: This work was supported by the National Natural Science Foundation of China (81770266), the "Six-one" Project for High-level Health Talents (No. LGY2016037).

\section{Footnote}

Reporting Checklist: The authors have completed the REMARK reporting checklist. Available at https://dx.doi. org/10.21037/atm-21-36

Peer Review File: Available at https://dx.doi.org/10.21037/ atm-21-36

Conflicts of Interest: All authors have completed the ICMJE uniform disclosure form (available at https://dx.doi. org/10.21037/atm-21-36). The authors have no conflicts of interest to declare.

Ethical Statement: The authors are accountable for all aspects of the work in ensuring that questions related to the accuracy or integrity of any part of the work are appropriately investigated and resolved. The study was conducted in accordance with the Declaration of Helsinki (as revised in 2013). This retrospective study was authorized by the Clinical Research Ethics Committee of the Affiliated Hospital of Nantong University, Jiangsu, China (No. 2017K025). Each patient received and signed written informed consent.

Open Access Statement: This is an Open Access article distributed in accordance with the Creative Commons Attribution-NonCommercial-NoDerivs 4.0 International License (CC BY-NC-ND 4.0), which permits the noncommercial replication and distribution of the article with the strict proviso that no changes or edits are made and the original work is properly cited (including links to both the formal publication through the relevant DOI and the license). See: https://creativecommons.org/licenses/by-nc-nd/4.0/.

\section{References}

1. Ferlay J, Shin H, Bray F, et al. Estimates of worldwide burden of cancer in 2008: GLOBOCAN 2008. Int J Cancer 2010;127:2893-917.

2. Chen $\mathrm{W}$, Zheng $\mathrm{R}$, Baade $\mathrm{P}$, et al. Cancer statistics in China, 2015. CA Cancer J Clin 2016;66:115-32.

3. Molina JR, Yang P, Cassivi SD, et al. Non-small cell lung cancer: epidemiology, risk factors, treatment, and 
survivorship. Mayo Clin Proc 2008;83:584-94.

4. Aberle DR, DeMello S, Berg CD, et al. Results of the two incidence screenings in the National Lung Screening Trial. New Engl J Med 2013;369:920-31.

5. Minguet J, Smith K, Bramlage P. Targeted therapies for treatment of non-small cell lung cancer--Recent advances and future perspectives. Int J Cancer 2016;138:2549-61.

6. Babon JJ, Stockwell D, DiRago L, et al. Membraneassociated RING-CH (MARCH) proteins down-regulate cell surface expression of the interleukin-6 receptor alpha chain (IL6R $\alpha$ ). Biochem J 2019;476:2869-82.

7. Bobby R, Robustelli P, Kralicek A, et al. Functional implications of large backbone amplitude motions of the glycoprotein 130-binding epitope of interleukin-6. Febs J 2014;281:2471-83.

8. Zhang JZ, Liu CM, Peng HP, et al. Association of genetic variations in IL-6/IL-6R pathway genes with gastric cancer risk in a Chinese population. Gene 2017;623:1-4.

9. Ehlers M, Grötzinger J, deHon F, et al. Identification of two novel regions of human IL-6 responsible for receptor binding and signal transduction. J Immunol 1994;153:1744-53.

10. Paonessa G, Graziani R, De Serio A, et al. Two distinct and independent sites on IL-6 trigger gp 130 dimer formation and signalling. Embo J 1995;14:1942-51.

11. Ozbek S, Grötzinger J, Krebs B, et al. The membrane proximal cytokine receptor domain of the human interleukin-6 receptor is sufficient for ligand binding but not for gp130 association. J Biol Chem 1998;273:21374-9.

12. Aasland D, Oppmann B, Grötzinger J, et al. The upper cytokine-binding module and the Ig-like domain of the leukaemia inhibitory factor (LIF) receptor are sufficient for a functional LIF receptor complex. J Mol Biol 2002;315:637-46.

13. Rokavec M, Öner M, Li H, et al. IL-6R/STAT3/ miR-34a feedback loop promotes EMT-mediated colorectal cancer invasion and metastasis. J Clin Invest 2014;124:1853-67.

14. Wei X, Wang C, Feng H, et al. Effects of ALOX5, IL6R and SFTPD gene polymorphisms on the risk of lung cancer: A case-control study in China. Int Immunopharmacol 2020;79:106155.

15. Yuquan B, Hexiao T, Laiyi $W$, et al. Interaction between epidermal growth factor receptor and interleukin-6 receptor in NSCLC progression. J Cell Biochem 2019;120:872-81.

16. Alberti C, Pinciroli P, Valeri B, et al. Ligand-dependent EGFR activation induces the co-expression of IL-6 and
PAI-1 via the NFkB pathway in advanced-stage epithelial ovarian cancer. Oncogene 2012;31:4139-49.

17. Wehbe H, Henson R, Meng F, et al. Interleukin-6 contributes to growth in cholangiocarcinoma cells by aberrant promoter methylation and gene expression. Cancer Res 2006;66:10517-24.

18. Liu X, Zhang A, Xiang J, et al. miR-451 acts as a suppressor of angiogenesis in hepatocellular carcinoma by targeting the IL-6R-STAT3 pathway. Oncol Rep 2016;36:1385-92.

19. Xu B, Chen Q, Yue C, et al. Prognostic value of IL6R mRNA in lung adenocarcinoma and squamous cell carcinoma. Oncol Lett 2018;16:2935-48.

20. Detterbeck FC, Chansky K, Groome P, et al. The IASLC Lung Cancer Staging Project: Methodology and Validation Used in the Development of Proposals for Revision of the Stage Classification of NSCLC in the Forthcoming (Eighth) Edition of the TNM Classification of Lung Cancer. J Thorac Oncol 2016;11:1433-46.

21. Tomczak K, Czerwińska P, Wiznerowicz M. The Cancer Genome Atlas (TCGA): an immeasurable source of knowledge. Contemp Oncol (Pozn) 2015;19:A68-77.

22. Liu K, Wang S, Liu Y, et al. Overexpression of MYCN promotes proliferation of non-small cell lung cancer. Tumour Biol 2016;37:12855-66.

23. Ji L, Li H, Gao P, et al. Nrf2 pathway regulates multidrugresistance-associated protein 1 in small cell lung cancer. Plos One 2013;8:e63404.

24. Gentles AJ, Newman AM, Liu CL, et al. The prognostic landscape of genes and infiltrating immune cells across human cancers. Nat Med 2015;21:938-45.

25. Yang L, Ying S, Hu S, et al. EGFR TKIs impair lysosomedependent degradation of SQSTM1 to compromise the effectiveness in lung cancer. Signal Transduct Target Ther 2019;4:25.

26. Bowker N, Shah R, Sharp S, et al. Meta-analysis investigating the role of interleukin-6 mediated inflammation in type 2 diabetes. Ebiomedicine 2020;61:103062.

27. Zhang C, Wu Z, Li J, et al. Cytokine release syndrome in severe COVID-19: interleukin-6 receptor antagonist tocilizumab may be the key to reduce mortality. Int J Antimicrob Agents 2020;55:105954.

28. Hashwah H, Bertram K, Stirm K, et al. The IL-6 signaling complex is a critical driver, negative prognostic factor, and therapeutic target in diffuse large B-cell lymphoma. Embo Mol Med 2019;11:e10576.

29. Schmidt-Arras D, Rose-John S. IL-6 pathway in the 
liver: From physiopathology to therapy. J Hepatol 2016;64:1403-15.

30. Zhou Y, Xia Z, Cheng Z, et al. Inducible microRNA590-5p inhibits host antiviral response by targeting the soluble interleukin-6 (IL6) receptor. J Biol Chem 2018;293:18168-79.

31. Rose-John S, Scheller J, Elson G, et al. Interleukin-6 biology is coordinated by membrane-bound and soluble receptors: role in inflammation and cancer. J Leukocyte Biol 2006;80:227-36.

32. Tsukamoto H, Fujieda K, Hirayama M, et al. Soluble IL6R Expressed by Myeloid Cells Reduces Tumor-Specific Th1 Differentiation and Drives Tumor Progression. Cancer Res 2017;77:2279-91.

33. Yang X, Hao H, Xia Z, et al. Soluble IL-6 Receptor and IL-27 Subunit p28 Protein Complex Mediate the Antiviral Response through the Type III IFN Pathway. J Immunol 2016;197:2369-81.

34. Rosa M, Chignon A, Li Z, et al. A Mendelian randomization study of IL6 signaling in cardiovascular diseases, immune-related disorders and longevity. Npj Genom Med 2019;4:23.

35. Wang Y, Hu H, Wu J, et al. The IL6R gene polymorphisms are associated with sIL-6R, IgE and

Cite this article as: Sun G, Wang T, Shi M, Zhou H, Wang J, Huang Z, Zhang H, Shi J. Low expression of IL6R predicts poor prognosis for lung adenocarcinoma. Ann Transl Med 2021;9(13):1057. doi: 10.21037/atm-21-36 lung function in Chinese patients with asthma. Gene 2016;585:51-7.

36. Ahmed S, Hussain S, Ammar A, et al. Interleukin 6 Receptor (IL6-R) Gene Polymorphisms Underlie Susceptibility to Rheumatoid Arthritis. Clin Lab 2017;63:1365-9.

37. Müller-Newen G, Küster A, Hemmann U, et al. Soluble IL-6 receptor potentiates the antagonistic activity of soluble gp130 on IL-6 responses. J Immunol 1998;161:6347-55.

38. Høgdall D, O'Rourke C, Dehlendorff C, et al. Serum IL6 as a Prognostic Biomarker and IL6R as a Therapeutic Target in Biliary Tract Cancers. Clin Cancer Res 2020;26:5655-67.

39. Wang K, Kim M, Di Caro G, et al. Interleukin-17 receptor a signaling in transformed enterocytes promotes early colorectal tumorigenesis. Immunity 2014;41:1052-63.

40. McAllister F, Bailey J, Alsina J, et al. Oncogenic Kras activates a hematopoietic-to-epithelial IL-17 signaling axis in preinvasive pancreatic neoplasia. Cancer cell 2014;25:621-37.

41. Furuta S, Jeng Y, Zhou L, et al. IL-25 causes apoptosis of IL-25R-expressing breast cancer cells without toxicity to nonmalignant cells. Sci Transl Med 2011;3:78ra31. 

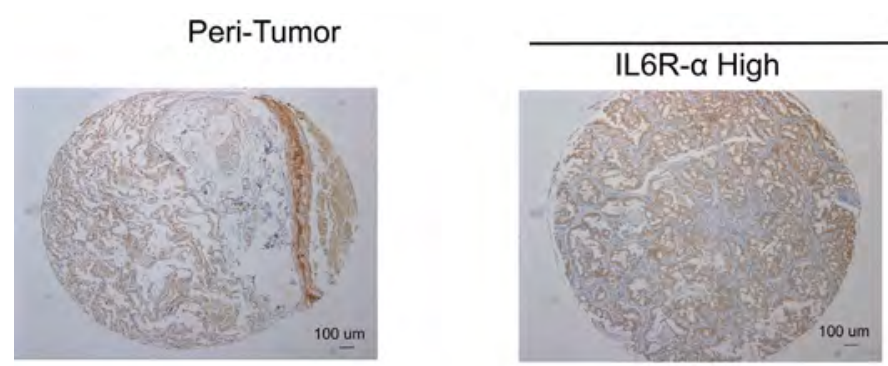

Tumor
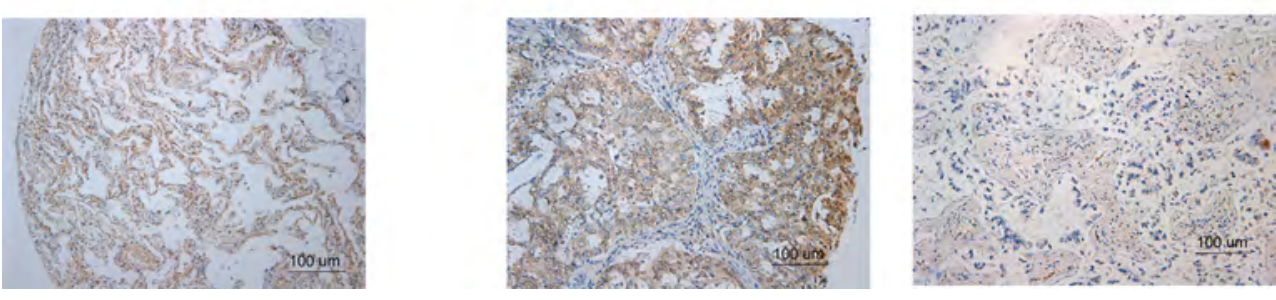

Figure S1 Representative images of interleukin 6 receptor (IL6R) IHC were taken at 5× (top) and 20× (bottom) magnifications. The scale bars are marked in the lower right corner of the image. 

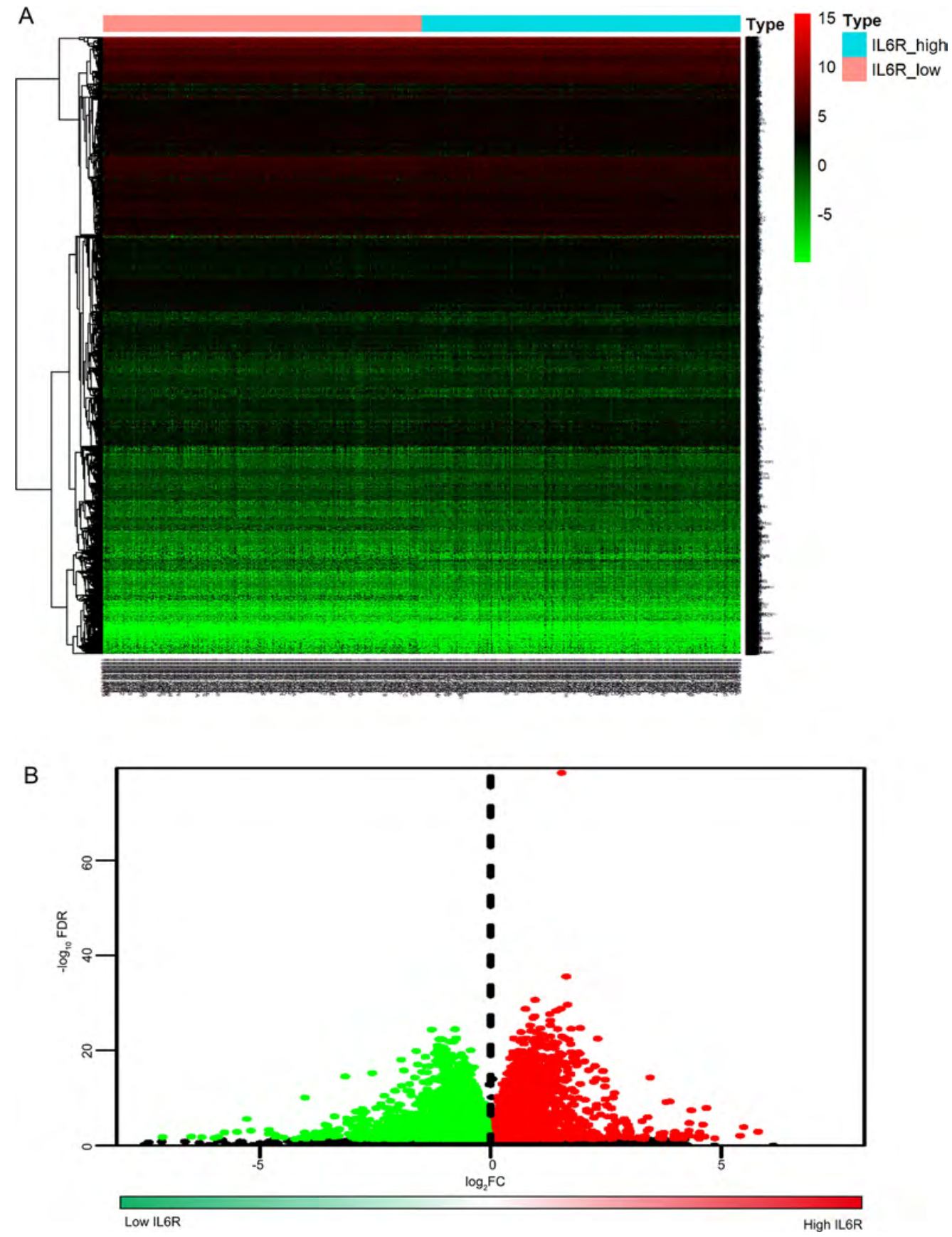

Figure S2 Heat map and volcano map of differential genes between high and low interleukin 6 receptor (IL6R) expression groups. (A) Heat map; (B) Volcano map. 


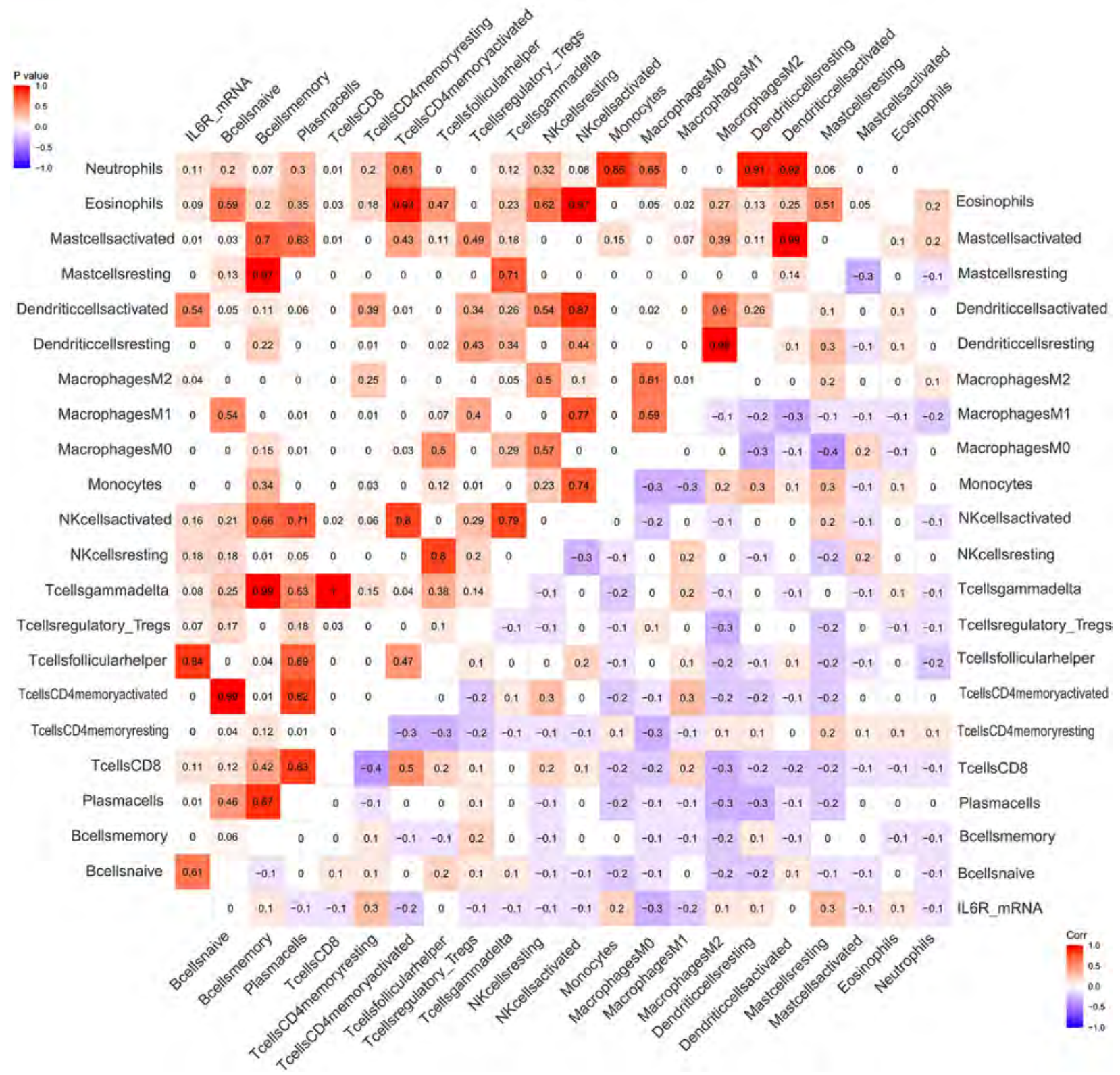

Figure S3 Heat map of the correlation of 21 immune infiltrating cells. 